\title{
Desempenho inicial de arroz irrigado decorrentes da aplicação de fertilizantes na seletividade de herbicidas
}

\section{Performance irrigated rice initial resulting from fertilizer application in herbicide selectivity}

\author{
Thais D'Avila Rosa ${ }^{1}$, Diogo Balbé Helgueira ${ }^{2}$, Daniele Brandstetter Rodrigues ${ }^{3}$, Diego Cardoso de Medeiros ${ }^{4}$, \\ Luis Antonio de Avila
}

\begin{abstract}
Resumo: O arroz, alimento apreciado na mesa do consumidor em todo mundo, possui amplo espectro produtivo no Brasil, devido às condições ideais de clima e solo encontrados no país, as quais possibilitam produção tanto em terras altas como em terras baixas, garantindo o abastecimento do mercado interno e externo. O presente estudo objetivou avaliar os efeitos decorrentes da aplicação de fertilizantes no desempenho inicial da cultura do arroz irrigado e na seletividade a herbicidas. O experimento foi conduzido em casa de vegetação, nas dependências da Faculdade de agronomia Eliseu Maciel - IES, Cidade (UF). O delineamento utilizado foi inteiramente casualizado, disposto em esquema fatorial ( 3 x 3$)+1$, com 4 repetições, onde o fator A corresponde aos fertilizantes (Biozyme $\mathrm{TF}^{\circledR}$, GreenFactor ${ }^{\circledR}$ e Pilatus ${ }^{\circledR}$ ) e o fator B aos herbicidas (cialofope-butil, bispiribaque-sódico e profoxidim) registrados para a cultura do arroz irrigado, além do tratamento controle, sem aplicação de fertilizante e sem aplicação de herbicidas. Avaliou-se o comprimento de parte aérea aos 7 e 21 dias após (DAH), massa da matéria seca aos $21 \mathrm{DAH}$, fitotoxicidade aos 7, 14 e $21 \mathrm{DAH}$ e teor de clorofila através de leituras com clorofilômetro. A partir dos resultados obtidos, observou-se que a aplicação dos fertilizantes não influenciou no desenvolvimento inicial da cultura do arroz e na seletividade dos herbicidas aplicados. Em relação à avaliação de fitotoxicidade, o profoxidim causou os maiores índices.
\end{abstract}

Palavras-chave: clorofilômetro; fitotoxicidade; Oryza sativa L,

\begin{abstract}
Rice, food enjoyed at the dinner table in the world, has extensive production spectrum in Brazil, due to the ideal conditions of climate and soil found in the country, which enable production of both upland and lowland, ensuring market supply internal and external. This study aimed to evaluate the effects of the application of fertilizers in the initial performance of irrigated rice and selectivity to herbicides. The experiment was conducted in a greenhouse on the premises of Agronomy Faculty Eliseu Maciel - IES, City (UF). The design was completely randomized, arranged in a factorial scheme (3 x 3) + 1, with 4 replications, where factor A corresponds to fertilizers (Biozyme TF®, GreenFactor® and Pilatus ${ }^{\circledR}$ ) and factor B herbicide (cyhalofop butyl, bispyribac-sodium and profoxidim) registered for irrigated rice in addition to the control treatment without fertilizer application and without application of herbicides. We evaluated the shoot length at 7 and 21 days (DAH), dry matter to $21 \mathrm{DAH}$, phytotoxicity at 7, 14 and $21 \mathrm{DAH}$ and chlorophyll content through readings with chlorophyll. From the results, it was observed that the application of fertilizers did not affect the early development of rice cultivation and selectivity of herbicides applied. Regarding the assessment of phytotoxicity, the profoxidim caused the highest rates.
\end{abstract}

Key words: chlorophyll; phytotoxicity,; Oryza sativa L.

\footnotetext{
*Autor para correspondência

Recebido para publicação em 15/08/2016; aprovado em 29/09/2016

${ }^{1}$ Doutoranda do PPG em C\&T de Sementes, FAEM/UFPel, Pelotas-RS, thais.d.rosa@ hotmail.com

${ }^{2}$ Universidade Federal de Pelotas , E-mail: diogobalbe@gmail.com

Mestre em Fitossanidade - Herbologia, Pelotas-RS.

${ }^{3}$ Mestrando no PPG em C\&T de Sementes, FAEM/UFPel, Pelotas-RS.

${ }^{4}$ Professor da Faculdade de Agronomia Eliseu, FAEM/UFPel.
} 


\section{INTRODUÇÃO}

$\mathrm{O}$ arroz está entre os cereais mais consumidos do mundo (CONAB, 2014), as condições favoráveis de solo e clima, fazem do Brasil o único país que consegue a efetividade na produção tanto em terras altas, como em terras baixas com irrigação. Diante, desse cenário, a produção da cultura leva a garantia do abastecimento da população do país e a exportação dos excedentes (FERREIRA2014).

Os fertilizantes, adubos, agrotóxicos, considerados insumos agrícolas, devido a evolução das técnicas utilizadas na produção, vêm abrangendo seu nicho no mercado, principalmente em culturas de importância econômica Dentre esses insumos, pode-se mencionar o Green Factor ${ }^{\circledR}$, produto considerado organomineral classe A registrado no Ministério da Agricultura Pecuária e Abastecimento (MAPA) decorrentes do nível zero de toxidade dermal e oral e por não conter sódio (MAPA, 2014).

O Green Factor ${ }^{\circledR}$ é um fertilizante produzido a partir do agrupamento de íons múltiplos que formam ligações com enzimas, através da sequência de aminoácidos dos peptídeos, transformando a matéria inorgânica em orgânica, possui na sua formulação fósforo, cobre e carbono orgânico (LBE, 2014). Na planta, o produto ativa a capacidade de absorver nitrogênio do ar, o que substitui a aplicação de nitrogênio em cobertura e, da bactéria usada como inoculante, na cultura da soja, GUERRA (2014), sendo sua aplicação no solo ou via foliar (LBE, 2014)

O nitrogênio, é elemento presente na maioria desses produtos, sendo que para o crescimento e desenvolvimento das plantas, o nitrogênio é considerado de suma importância pois participa diretamente do processo fotossintético das plantas. Especificamente na cultura do arroz, em relação a sua produtividade, a disponibilidade desse elemento e sua ligação com o acréscimo de outros componentes de rendimento são avaliados como fatores que influenciam no processo (FAGERIA; STONE, 2003).

Em um sistema de cultivo, as análises do teor de matéria orgânica do solo (MOS), são determinantes em grande parte, para a recomendação da adubação nitrogenada da cultura, esse parâmetro serve como indicativo da disponibilidade desse elemento, durante o ciclo de uma cultura (ARGENTA, 2001). Entretanto, em solo alagado, como ocorre com a cultura do arroz irrigado, onde o método de irrigação é por inundação, essas analises se tornam insuficientes, não sendo estimado um índice preciso para a avaliar a disponibilidade desse elemento no solo (SCIVITTARO; MACHADO, 2004).

Este fator pode estar conexo às alterações na dinâmica do nitrogênio devido ao processo de estresse hídrico e consequentemente, sua diminuição no solo. Em condições de ausência de oxigênio, a decomposição da matéria orgânica do solo é praticamente nula, devido anaerobiose e redução de microorganismos capazes de executar tal processo (SOUSA et al., 2004).

Segundo Datta e Broadbent (1988) de 20 a $40 \%$ do nitrogênio presente nos fertilizantes são utilizados pela cultura do arroz em solo alagado, essa porcentagem é considerada baixa, comparada a eficiência e solo sem alagamento.

Uma das causas dessa baixa eficiência são decorrentes das perdas desse elemento, como por exemplo volatilização, imobilização e lixiviação (FAGERIA; STONE,
2003). Diante do exposto, o objetivo do trabalho é avaliar as respostas no desenvolvimento inicial da cultura do arroz irrigado IRGA 424, decorrentes da aplicação de fertilizantes e herbicidas.

\section{MATERIAL E MÉTODOS}

O estudo foi conduzido entre os meses de setembro a novembro de 2014 em casa de vegetação localizado no Município do Capão do Leão, RS, com latitude de $31^{\circ} 47^{`} \mathrm{~S}$, longitude de $52^{\circ} 25^{\circ} \mathrm{W}$ e 13 metros de altitude, sendo a temperatura média nos meses da condução do experimento de $30^{\circ} \mathrm{C}$. O experimento foi realizado Departamento de Fitossanidade, da Faculdade de Agronomia Eliseu Maciel (FAEM), Universidade Federal de Pelotas (UFPel), localizada no município de Capão do Leão, RS.

O delineamento utilizado foi inteiramente casualizado, arranjado em esquema fatorial $3 \times 3$, com quatro repetições. No fator A são considerado os fertilizantes e no fator B são alocados os herbicidas utilizados na cultura do arroz. Foram utilizados tratamentos testemunhas, sem aplicação de fertilizante e sem aplicação de herbicidas.

Os fertilizantes e suas respectivas doses que compunham o fator A foram: Biozyme $\mathrm{TF}^{\circledR}\left(250 \mathrm{~mL} / \mathrm{ha}^{-1}\right)$, Green Factor $^{\circledR}\left(150 \mathrm{~mL} / \mathrm{ha}^{-1}\right)$ e Pilatus ${ }^{\circledR}\left(150 \mathrm{~mL} / \mathrm{ha}^{-1}\right)$. Os tratamentos com herbicidas correspondentes ao fator $\mathrm{B}$, conforme a tabela 1 .

Tabela 1- Herbicidas registrados e recomendados para o controle de plantas daninhas na cultura do arroz irrigado no RS e SC. FAEM/UFPel, Capão do Leão, RS

\begin{tabular}{ccc}
\hline Herbicidas & $\left.\begin{array}{c}\text { DRp.c* } \\
(\mathrm{g} \text { i.a. ha }\end{array}{ }^{-1}\right)$ & Época de Aplicação \\
\hline Controle & 0 & 0 \\
Cialofope-butil & 315 & Pós-emergência \\
Bispiribaque-sódico & 50 & Pós-emergência \\
Profoxidim & 170 & Pós-emergência \\
\hline
\end{tabular}

*DRp.c: Dose de Resistro p.c

Foram utilizadas sementes de arroz cultivar IRGA 424 , as unidades experimentais foram constituídas de caixas de plástico com capacidade de $20 \mathrm{~L}$ preenchidas com $15 \mathrm{~kg}$ de solo coletado do horizonte A de um Planossolo Háplico Eutrófico solódico, seco em ar livre e peneirado, em peneira de malha 2,0 $\mathrm{mm}$. Em cada unidade experimental foram semeadas aproximadamente 60 sementes de arroz cultivar IRGA 424, distribuídas em três linhas.

$\mathrm{O}$ tratamento com o produto Biozyme $\mathrm{TF}^{\circledR}$ foi realizado diretamente nas sementes, segundo as recomendações da bula, com válvula pressurizada, 24 horas anterior à semeadura sendo colocadas em sacos plásticos com capacidade para cinco litros, utilizando um $1 \mathrm{~kg}$ de sementes por saco.

A aplicação dos fertilizantes Green Factor $^{\circledR} \mathrm{e}$ Pilatus ${ }^{\circledR}$ foi realizada com o auxílio de um borrifador, na dose de $150 \mathrm{ml} \mathrm{ha}^{-1}$, sendo aplicados na base das plantas de arroz, um dia antes da aplicação dos herbicidas.

A aplicação dos herbicidas foi realizada com auxílio de um pulverizador costal pressurizado com $\mathrm{CO}_{2}$, equipado com barra de quatro bicos de jato plano em leque, série 11002 , espaçadas $50 \mathrm{~cm}$, calibrado para aplicar volume de calda 
de $150 \mathrm{~L} \mathrm{ha}^{-1}$. No dia seguinte a aplicação, as caixas foram inundadas, mantendo a lâmina d'água de $80 \mathrm{~mm}$.

A fitotoxicidade foi avaliada aos 7, 14 e 21 dias após a aplicação dos herbicidas (DAH), visualmente, através da atribuição de notas baseadas em escala percentual de 0 a $100 \%$, onde zero corresponde à ausência de injúrias e 100\% corresponde à morte das plantas, segundo a proposta da Sociedade Brasileira da Ciência das Plantas Daninhas (1995).

Avaliou-se o comprimento da parte aérea aos 14 dias após a aplicação dos herbicidas sendo utilizadas quatro plântulas por tratamento, com auxílio de uma régua milimetrada. Os dados foram submetidos à análise de variância e quando significativos pelo teste $F(p \leq 0,05)$, às médias comparadas pelo teste de Tukey $(\mathrm{p} \leq 0,05)$. A variável porcentagem de germinação foi transformada pela equação $\mathrm{yt}=\sqrt{\mathrm{y}}+1$ para fins de análises estatísticas.

\section{RESULTADOS E DISCUSSÃO}

Os resultados da análise de variância demonstraram $\mathrm{O}$ volume de calda utilizado foi de $1,5 \mathrm{~L} 100 \mathrm{~kg}^{-1}$ de semente, para o tratamento controle foi utilizado apenas água que não ocorreu interação entre os fatores fertilizantes e herbicidas, porém foi verificado diferença significativa entre os herbicidas aplicados.

Aos 14 DAH o comprimento da parte aérea das plantas de arroz, não apresentou diferença estatística para a avaliação em relação aos fertilizantes aplicados. Mesmo não havendo diferença estatística entre os fatores, cabe salientar que as cultivares de arroz comportam-se diferentemente em relação a eficiência ao uso do nitrogênio, esse fator foi observado por Dos Passos (2015), que avaliou a eficiência do uso de nitrogênio em diferentes genótipos de arroz. O mesmo autor descreve que dentre as cultivares mais responsivas ao uso do elemento está a cultivar IRGA 424.

Tabela 2. Comprimento da parte aérea (CPA) na cultura do arroz irrigado, em função dos tratamentos herbicidas aos 14 dias após a aplicação. FAEM/UFPel - Capão do Leão, RS

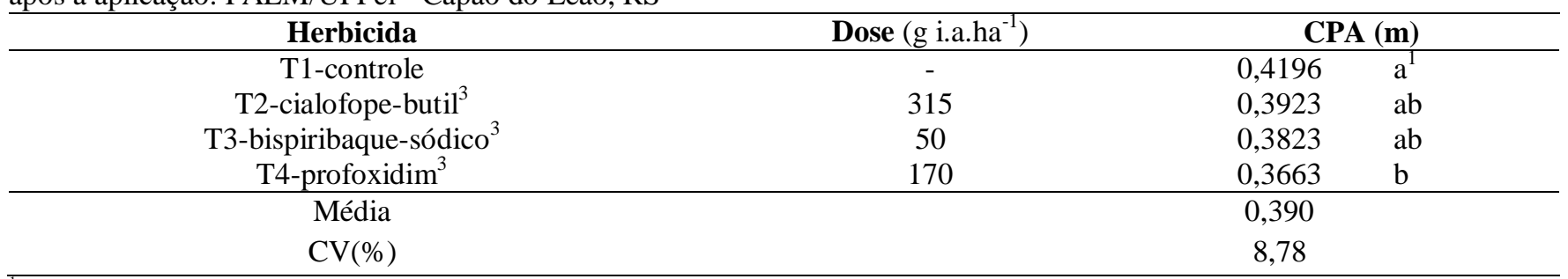

${ }^{\mathrm{T}}$ Médias com letras distintas na coluna diferem pelo teste de Tukey $(\mathrm{p} \leq 0,05)$.

${ }^{2}$ Dias após a aplicação dos herbicidas.

${ }^{3}$ Aplicação em pós-emergência com 3 a 4 folhas $\left(V_{3}-V_{4}\right)$.

Os herbicidas cialofope-butil e bispiribaque-sódico, não diferiram do tratamento controle e do tratamento com o herbicida profoxidim. Diante da aplicação do herbicida profoxidim a cultura apresentou comprimentos inferiores de parte aérea das plantas de arroz aos $14 \mathrm{DAH}$, comparado ao tratamento controle (Tabela 2).

Com relação a aplicação de herbicidas, um dos agravantes é a possibilidade desses produtos causarem fitotoxicidade à cultura $\mathrm{A}$ seletividade de herbicidas ao arroz pode variar de acordo com uma série de condições abióticas, como por exemplo luminosidade e temperatura (PETTER et al., 2011).

Trabalhos que avaliem os efeitos da aplicação de herbicidas na cultura do arroz são escassos, contudo muitos trazem os efeitos da aplicação no controle de plantas daninhas, principalmente quando se trata de alternativas no controle de plantas daninhas resistentes.

$\mathrm{Na}$ avaliação de fitotoxicidade houve diferença estatística entre os herbicidas para os três momentos de avaliação (Tabela 3). Na cultura do arroz, a época de aplicação influencia na seletividade do herbicida, Veske et al., (2016), avaliando a aplicação dos herbicidas bispiribaquesódico e penoxsulam, observaram que a aplicação do bispiribaque-sódico na época recomendada não acarretou em perdas de produtividade para a cultura.

$\mathrm{Na}$ avaliação de fitotoxicidade aos $7 \mathrm{DAH}$, houve diferença entre os herbicidas em que as plantas submetidas à aplicação de profoxidim, apresentaram maior índice de fitotoxicidade diferindo dos outros tratamentos. Os tratamentos com cialofope-butílico e bispiribaque-sódico não diferiram entre si (Tabela 3).

Tabela 3. Fitotoxicidade na cultura do arroz irrigado, em função dos tratamentos herbicidas aos sete, 14 e 21 dias após a aplicação dos herbicidas. FAEM/UFPel - Capão do Leão, RS

\begin{tabular}{cccc}
\hline & \multicolumn{3}{c}{ Fitotoxicidade (\%) } \\
\cline { 2 - 4 } Herbicida & & 14 DAH & 21 DAH \\
\hline T1-controle & 07 DAH $^{2}$ & $0,0 \mathrm{c}$ & $0,0 \mathrm{c}$ \\
T2-cialofope-butil $^{3}$ & $0,0 \mathrm{c}^{1}$ & $2,22 \mathrm{c}$ & $10,0 \mathrm{~b}$ \\
T3-bispiribaque-sódico $^{3}$ & $29,05 \mathrm{~b}$ & $17,22 \mathrm{~b}$ & $13,33 \mathrm{ab}$ \\
T4-profoxidim $^{3}$ & $29,72 \mathrm{~b}$ & $31,66 \mathrm{a}$ & $20,55 \mathrm{a}$ \\
\hline Média & $52,50 \mathrm{a}$ & 12,77 & 10,97 \\
\hline CV $(\%)$ & 27,69 & 23,5 & 35,21
\end{tabular}

${ }^{\mathrm{T}}$ Médias com letras distintas na coluna diferem pelo teste de Tukey $(\mathrm{p} \leq 0,05)$.

${ }^{2}$ Dias após a aplicação dos herbicidas.

${ }^{3}$ Aplicação em pós-emergência com 3 a 4 folhas $\left(V_{3}-V_{4}\right)$. 
Os resultados corroboram com os encontrados por Costa et. al., (2013), onde foram observados maiores valores de fitotoxicidade aos 7 DAH com aplicação de profoxidim em relação aos outros herbicidas, porém o efeito fitotóxico tornou-se menos acentuado aos 14 e 21 dias após a aplicação dos herbicidas.

$\mathrm{Na}$ avaliação aos $14 \mathrm{DAH}$, foi observado diferença na avaliação de fitotoxicidade para os herbicidas aplicados (Tabela 3). O tratamento com o herbicida profoxidim proporcionou maior nível fitotóxico quando comparado ao controle, seguido pelo tratamento com o herbicida bispiribaque-sódico, Galon et al., (2015) avaliando a eficácia e fitotoxicidade de diferentes herbicidas aplicados na cultura do trigo para controle de plantas daninhas, observaram que os herbicidas imazethapyr + imazapic, clomazone e imazapic + imazapyr apresentaram maior fitotoxicidade na cultura.

Aos 21 DAH, os tratamentos cialofope-butil foi o tratamento que apresentou o menor valor de fitotoxicidade as plantas de arroz, não diferindo do tratamento com a aplicação de bispiribaque-sódico. Mesmo não expressivo, pois não diferiu do tratamento bispiribaque-sódico, o profoxidim ainda apresentou os maiores valores absolutos de fitotoxicidade no arroz.

Esse resultado também foi observado por Cassol et. al., (2013), onde a aplicação do herbicida profoxidim no arroz irrigado proporcionou aumento da fitotoxicidade em relação ao herbicida cialofope-butil, porém esse resultado não influenciou na sua produtividade.

Pode-se observar que ao longo das avaliações da fitotoxicidade foi reduzido em relação as primeiras avaliações (sete DAH), A capacidade de recuperação das plantas de arroz também pode ser observado por Webster e Masson, (2001) com aplicação de nicosulfuron e Levy Jr. et al., (2006) com aplicação do herbicida imazethapyr.

Ainda não está bem esclarecido na literatura, quais os aparatos que as plantas de arroz desempenham em relação a capacidade de recuperação, o que é verificado que essa capacidade dependerá da dose do herbicida utilizado, e das características da cultivar, sensível ou tolerante ao herbicida,

\section{CONCLUSÕES}

A aplicação de fertilizantes na cultura do arroz não influencia no desenvolvimento inicial e na sensibilidade da cultura do arroz irrigado a herbicidas. A aplicação do herbicida profoxidim apresentou o maior nível de fitotoxicidade na cultura, em todas as avaliações.

\section{REFERÊNCIAS}

ARGENTA, G.; SILVA, P. R. F.; BORTOLINI, C. G. Clorofila na folha como indicador do nível de nitrogênio em cereais. Ciência Rural, v.31, p.715-722, 2001.

CASSOL, G.V. AVILA, L. A.; ZEMOLIN, C. R.; PESTANA, R. R.; AGOSTINETTO, D. Eficácia de tratamentos herbicidas na cultura do arroz irrigado sob irrigação intermitente. In: VIII Congresso Brasileiro de Arroz Irrigado, 2013, Santa Maria, RS. Anais... Sta. Maria, SOSBAI, 2013, v.1, p.391-394, 2013.
COMPANHIA NACIONAL DE ABASTECIMENTO, CONAB, 2014 Acompanhamento da safra brasileira de grãos - Safra 2013/2014. Disponível em: <http://www.agricultura.gov.br/vegetal/culturas/arroz> Acesso em: Dezembro de 2014.

COSTA, C.C.; SEVERO, A. J.; SPAT, L. L.; DONELLES, S. H. B.; PERINI, F. L.; SQUASSONI, V. L. Efeito do herbicida metamifop no controle de Eragrostis lugens em arroz irrigado. Cbai 2013. Anais... 2013. Disponível em <http://www.cbai2013.com.br/cdonline/docs/trab-4136645.pdf> Acesso em: Novembro de 2014.

DATTA, S.K DE.; BROADBENT, F.E. Methodology for evaluating nitrogen utilization efficiency by rice genotypes. Agronomy Journal, v.80, p.793-798, 1988.

PASSOS, N. G. dos, DE SOUSA, S. A., LOPES, M. B. S., VARAVALLO, M. A., DE OLIVEIRA, T. C., \& FIDELIS, R. R. Eficiência no uso de nitrogênio em genótipos de arroz em solos de várzea tropical do Estado do Tocantins. Revista Agro@ mbiente On-line, v. 9, n. 1, p. 8-16, 2015.

FAGERIA, N.K.; STONE, L.F. Manejo do Nitrogênio. In: FAGERIA, N.K.; STONE, L.F.; SANTOS, A.B. dos. Manejo da Fertilidade do Solo para o Arroz Irrigado. Santo Antônio de Goiás: Embrapa Arroz e Feijão, 2003, p.51-94.

FERREIRA, C.M. 2014. Rede brasil arroz: transferência de tecnologia para a orizicultura brasileira. Embrapa Arroz e Feijão. Disponível em: <http://ainfo.cnptia.embrapa.br/digital/bitstream/item/104910/ 1/p1554.pdf> Acesso em: Outubro de 2014.

GALON, L.; CASTOLDI, C. T.; FORTE, C. T.; KUJAWISKI, R.; DE DAVID, F. A.; PERIN, G. F.; RADUNZ, L. L. Eficácia e fitotoxicidade de herbicidas aplicados para manejo de plantas daninhas infestantes do trigo. Revista Brasileira de Herbicidas, v. 14, n. 2, p. 128-140, 2015.

GUERRA, J. Universo Agro 2013. Disponível em: http://www.uagro.com.br/editorias/pesquisa-e tecnologia/biotecnologia/2013/08/05/chega-ao-mercadonovo-fertilizanteorganomineral.html> Acesso em: Novembro de 2014.

LEVY JR., R. J.; BOND, J. A., WEBSTER, E. P., GRIFFIN, J. L., \& LINSCOMBE, S. D. Effect of cultural practices on weed control and crop response in imidazolinone-tolerant rice. Weed Technology., v. 20, p. 249-254, 2006.

$\begin{array}{lcrr}\text { MINISTÉRIO DA AGRICULTURA, MAPA } & 2014 . \\ \begin{array}{l}\text { Fertilizantes. } \\ \text { http://www.agricultura.gov.br/vegetal/fertilizantes> }\end{array} & \text { Acesso }\end{array}$
em: Novembro de 2014.

PETTER F.A., ZUFFO A.M.; PACHECO L.P. Seletividade de herbicidas inibidores de ALS em diferentes estádios de desenvolvimento do arroz de terras altas. Pesquisa Agropecuária Tropical, v.41, p.408-414, 2011. 
SCIVITTARO, W.B.; MACHADO, M.O. Adubação e calagem para a cultura do arroz irrigado. In: GOMES, A. da S., MAGALHÃES JUNIOR, A. M. de (Org). Arroz irrigado no Sul do Brasil. Brasília-DF: Embrapa Informação Tecnológica, 2004. cap.9, p.259-303.

SOCIEDADE BRASILEIRA DA CIÊNCIA DAS PLANTAS DANINHAS - SBCPD. Procedimentos para a instalação, avaliação e análise de experimentos com herbicidas. Londrina: p. 45, 1995.

SOUSA, R.; CAMARGO F.A.O.; VAHL, L.C. Solos Alagados (Reações de redox). In: MEURER, E.J. (Ed.) Fundamentos da química do solo. 2. ed. Porto Alegre: Gênesis, 2004. p.208-237.

VENSKE, E. Schaedler, C. E., Ritter, R., Fin, S. S., Bahry, C. A., de Avila, L. A.; Zimmer, P. D. Seletividade de herbicidas sobre arroz irrigado em resposta à época de semeadura e redução da luminosidade em fases do desenvolvimento. Ceres, v. 63, n. 2, 2016.

WEBSTER, E. P.; MASSON, J. A. Acetolactate synthaseinhibiting herbicides on imidazolinona-tolerant rice. Weed Science., v. 49, p. 652-657, 2001. 